\title{
Fractions in Transrational Arithmetic
}

\author{
Jan A. Bergstra \\ j.a.bergstra@uva.nl, janaldertb@gmail.com \\ Informatics Institute, University of Amsterdam \\ Science Park, 904, 1098 XH, Amsterdam, The Netherlands
}

Submitted: 6 June 2019

Revised: 24 January 2020

\begin{abstract}
In transrational arithmetic each closed fraction may be written as a simple fraction. It is shown that unlike in involutive meadows, in transrational arithmetic open fractions cannot be written as a sum of simple fractions (i.e. fractions the numerator and denominator of which are polynomials). It is also not the case that each open fraction with a single variable can be written as a mixed fraction.
\end{abstract}

\section{Introduction}

Different approaches to the issue of division by zero exist, a survey of such options is presented in [4]. As it turns out different mechanisms to go about $1 / 0$ result in quite different properties of the collection of fractions. With $\mathbb{Q}_{\perp}^{d}, \mathrm{I}$ denote the common meadow of rational numbers, that is the rational numbers with divisive notation (using division rather than inverse) with division made total by returning an absorptive element, denoted with $\perp$, when dividing by 0 . An absorptive value is often referred to as an error element in abstract datatype theory with $\perp$ used as its preferred notation, while in transmathematics $\Phi$ is used as the preferred notation for an absorptive value. The theory of fractions in $\mathbb{Q}_{\perp}^{d}$ is almost trivial as each expression (open or closed) can be written equivalently in the form $P / Q$, with $P$ and $Q$ not involving division. $P / Q$ is a so-called simple fraction, so that simple fractions provide a universal format for open expressions in the common meadow of rational numbers.

In the meadow of rational numbers $\mathbb{Q}_{0}^{d}$, that is the version of the rational numbers with divisive notation where inverse is made total by adopting $x / 0=0$ the situation is quite different. Working over $\mathbb{Q}_{0}^{d}$, each expression can be written as a sum of simple fractions ([7]), but no bound on the number of summands may be imposed ([10]), while univariate expressions may be transformed into mixed fraction format ([6]). The question whether bivariate expressions can be written in mixed fraction format is open in the case of $\mathbb{Q}_{0}^{d}$.

(C) Jan A. Bergstra 2019

Licence CC BY-SA 4.0 
The objective of this paper is to investigate the properties of fractions for $\mathbb{Q}_{ \pm \infty, \Phi}^{d}$, the arithmetical datatype of transrational numbers with divisive notation. Definitions of the structure of transrational numbers date back to around 2000. In [1] a structure corresponding to the transrationals as specified below is discussed with the exception that Table 1 of [1] indicates that $x / y=(-x) /(-y)$ holds in general, which we will not adopt, with the understanding that $(-1) /(-0)=(-1) / 0=(-1) \cdot(1 / 0)=(-1) \cdot \infty=-\infty \neq \infty=1 / 0$ thus maintaining the familiar identity $x / y=x \cdot(1 / y)$. In [2] axioms for the extension of transrational to transreals are listed. In [2] inversive notation is used, that is only inverse appears and no mention is made of division. In the absence of division, that is when working with inverse only, there is no discrepancy about the definition of transrational arithmetic. Below we have taken the liberty to work with division as defined by $x / y=x \cdot y^{-1}$ and by reading $x^{-1}$ as $1 / x$. For a theory of fractions divisive notation is needed, and variations in the definition of division, given a fixed inverse, impact on the resulting properties of fractions.

We find that the picture concerning properties of fractions in transrational arithmetic is significantly more complex than in the case of the meadow $\mathbb{Q}_{0}^{d}$. We have obtained partial results only, however, as some key questions have been left open. Transrational numbers combine the idea that $1 / 0$ is not finite with the idea that non-finite values are signed against the background that division is total. Under these intuitively appealing constraints the design of the structure of transrationals seems to have no plausible alternative. If only for that reason $\mathbb{Q}_{ \pm \infty, \Phi}^{d}$ merits an effort of pure research.

In the setting of transrational arithmetic some transformations exist which simplify fractions to a moderate extent, for instance:

$$
\frac{x \cdot x \cdot x}{x \cdot x}=\frac{x \cdot x}{x}, \frac{x \cdot x}{x \cdot x \cdot x}=\frac{x}{x \cdot x}, \frac{1}{x}+\frac{1}{1-x}=\frac{1}{x \cdot(1-x)}
$$

The proof of these equations is immediate: only values $x-0, \infty,-\infty, 1$ need to be checked which is trivial for each of these equations.

As it turns out, we could not find any stronger and generally applicable transformation for simplifying the structure of fractions in the context of $\mathbb{Q}_{ \pm \infty, \Phi}^{d}$. We leave open the question whether or not such transformations exist. In Section 3 we will introduce fraction depth as a complexity measure on fractions. Fraction depth can be applied in any arithmetical datatype where it introduces a hierachy on fractions which may or may not be proper. We leave open the fraction hierarchy problem (3.1 below) for transrational arithmetic.

\subsection{Naturals and integers, assimilation and dis-assimilation}

We assume that the set of natural numbers, $|\mathbb{N}|$, and the set of integers, $|\mathbb{Z}|$, are known (given) as specific sets so that $|\mathbb{N}| \subseteq|\mathbb{Z}|$. In fact these sets serve as parameters of the discussion. We have not fixed signatures for underlying arithmetical datatypes $\mathbb{N}$ and $\mathbb{Z}$, and we will use constants zero and one, and as 
functions addition, opposite, subtraction, multiplication, and greatest common divisor.

Readers may already be worried about the notation used at this stage. Why not use $\mathbb{N}$ instead of $|\mathbb{N}|$ ? Following [3] (see also [17]) we will adopt the idea that there is no such thing as "a natural number". Instead of determining which entities are natural numbers one may determine which structures are natural number structures, and then choose a preferred candidate from a plurality of options.

But at the same time, there is no such thing as "the signature of natural numbers" which may reliably serve as a predetermined prerequisite for the identification of natural number structures and for the selection of a preferred one of those. Determination of a signature for $\mathbb{N}$ is as much a matter of choice as is the determination of the class of abstract entities which one intends to consider natural numbers. The idea of the notation used is that $|\mathbb{N}|$ is a fixed but arbitrary choice of a countable infinite set, in some acceptable set theory, which serves as the collection of natural numbers, and that $\mathbb{N}$ is an arithmetical datatype the signature of which is arbitrary but fixed while including the constants and functions mentioned above. Moreover and similarly $|\mathbb{Z}|$ is a chosen superset of $|\mathbb{N}|$. Further we do not distinguish between formal names of constants and functions and the ordinary descriptions of these. Although assuming that 0 serves as the zero in $|\mathbb{N}|$, it is common practice in logic to have $\underline{0}$ as a constant in the formal language about $\mathbb{N}$ so that 0 is the interpretation of $\underline{0}$. Unfortunately the distinction between 0 and $\underline{0}$, however useful it may be in logic, constitutes an unpleasant, if not unworkable overhead in ordinary mathematical practice. For that reason we will do without $\underline{0}$ and we prefer to apply what is called explicit assimilation in [20]: aware of the fact that a distinction between 0 and $\underline{0}$ might be made, the two are assimilated and the distinction is not made. If it happens to be necessary to make a distinction between the two then explicit dis-assimilation may take place. Dis-assimilation can go either way: to introduce $\underline{0}$ as a formal counterpart of 0 , or to consider 0 as a syntactic entity rather than as a semantic entity and to introduce $\hat{0}$ as a semantic counterpart to the 0 being used.

Assimilation as applied in this case indicates that at the level of $\mathbb{N}$ and $\mathbb{Z}$ no distinction between syntax and semantics is made. That is a choice which

may be reversed at any time. Further we refer to the collection of constants and functions that are used for $\mathbb{N}$ and $\mathbb{Z}$ as a catalogue rather than a signature, thereby indicating that no syntactic or semantic bias is supposed to be present.

\subsection{Transrational arithmetic: fractions and flat forms}

We assume that $\mathbb{Q}$ is chosen to be a specific instance of the isomorphism class of ring of rational numbers, denoting with $|\mathbb{Q}|$ its domain. The domain of $\mathbb{Q}$ consists of pairs $(n, m)$ with, $n$ an integer, $m$ a positive integer, and $\operatorname{gcd}(n, m)=1$. The constants 0 and 1 are identified with the pairs $(0,1)$ and $(1,1)$ respectively.

We have developed a description of transrational arithmetic, up to isomorphism, by means of a term rewrite system involving infinitely many auxiliary 
constants in [4]. These constants coincide with the pairs $(n, m)$ mentioned above. The transrationals may be characterised as the most plausible structure which extends the domain $|\mathbb{Q}|$ of $\mathbb{Q}$ with positive infinity $(\infty)$ and negative infinity $(-\infty)$ and an entity called nullity $(\Phi)$ so that $1 / 0=\infty, 1 / \infty=1 /(-\infty)=$ $0,-(\infty)=-\infty, 0 \cdot \infty=0 \cdot(-\infty)=\infty+(-\infty)=\Phi$, where $\Phi$ serves as an error value propagating through all operations $(\Phi+x=\Phi \cdot x=-\Phi=\Phi$ etc.) Now $\left|\mathbb{Q}_{ \pm \infty, \Phi}\right|=|\mathbb{Q}| \cup\{\infty,-\infty, \Phi\}$. The elements of the domain of $\mathbb{Q}_{ \pm \infty, \Phi}$ which are in the domain of $\mathbb{Q}$ are called rational values, the other three elements are non-rational values. Non-rational values are to be distinguished from irrational values such as $\sqrt{2}$ that may exist in an algebraic extension of the transrational numbers.

Following [10] a fraction is defined as a particular kind of expression:

Definition 1.1. A fraction is an expression with division as the leading function symbol, that is an expression of the form $P / Q$ with $P$ and $Q$ expressions over the signature of so-called divisive meadows (zero, one, plus, opposite, minus, multiplication, division) or some extension thereof.

We mention that in the presence of inverse an expression of the form $P^{-1}$ is not considered a fraction. For a fraction $P / Q, P$ is called its numerator and $Q$ is called its denominator. The value of the fraction $P / Q$ in an arithmetical datatype is called the quotient of $P$ and $Q$. And, by consequence, rather than speaking of a field of fractions we prefer to speak of a field of quotients.

Definition 1.2. $P / Q$ is a simple fraction if $P$ and $Q$ are not fractions and do not have fractions as subterms.

Definition 1.3. A flat form is an expression which is not a fraction and which has no fractions as subterms.

Thus a simple fraction is a fraction made up of two flat forms. In this paper the signature of meadows $\Sigma_{\mathrm{Md}}$ is extended with three constants: $+\infty,-\infty$, and $\Phi$, thus obtaining the signature of transrationals $\Sigma_{\mathrm{Md}, \pm \infty, \Phi}$. We will use $P / Q$ and $\frac{P}{Q}$ as synonyms. Now one may view $\infty$ as an abbreviation of $1 / 0,-\infty$ as an abbreviation of $(-1) / 0$, and $\Phi$ as an abbreviation of $0 / 0$, but that perspective is immaterial for a theory of fractions. Because $\infty$ is a constant the fraction $0 / \infty$ is a simple fraction, whereas $0 /(1 / 0)$ is not a simple fraction, in spite of the fact that $\infty=1 / 0$. It transpires that the presence of new constants for values which can be expressed in terms of the signature of meadows is especially relevant for the development of a theory of fractions. A fraction is closed if it contains no variables, otherwise it is open. The following observation is an immediate consequence of the definition of transrationals.

Proposition 1.1. For each closed expression $t$ over signature $\Sigma_{\mathrm{Md}, \pm \infty, \Phi}$ there is a simple closed fraction $P / Q$ over signature $\Sigma_{\mathrm{Md}, \pm \infty, \Phi}$ such that $\mathbb{Q}_{ \pm \infty, \Phi}=$ $t=P / Q$. Moreover for this simple fraction it may be required that $Q$ is made up from one, addition and multiplication only (so that it can not be equal to 0 , $\infty,-\infty$ or $\Phi)$. 
The following fact is immediate by induction on the structure of flat forms.

Proposition 1.2. In transrational arithmetic any closed flat form $P$ over signature $\Sigma_{\mathrm{Md}, \pm \infty, \Phi}$ which contains $\infty$ as a subterm or contains $-\infty$ as a subterm has a value in $\{\infty,-\infty, \Phi\}$.

We recall that $\mathbb{Q}_{0}$ is the structure obtained from the rational numbers (with the signature of rings) by expanding the structure with inverse and division, both made total in the simplest possible manner, by setting $1 / 0=0 . \mathbb{Q}_{0}$ is a meadow, and as inverse is an involution in $\mathbb{Q}_{0}$ it is called an involutive meadow. In [7] it is shown that in any meadow, in particular in $\mathbb{Q}_{0}$, each fraction can be written as a sum of simple fractions. The same is not true, however, for transrational arithmetic, and the proof of that fact is the main result of this paper. The following immediate consequence of Proposition 1.1 will be used in the sequel.

Proposition 1.3. Let $R \equiv P_{1} / Q_{1}+\ldots+P_{n} / Q_{n}$ be a sum of simple fractions with variables among $x_{1}, \ldots, x_{k}$. If, in transrational arithmetic, for each $i \in$ $\{1, \ldots, n\}$ there are closed integer expressions $h_{j}, j \neq i, 1 \leq j \leq k$ such that $R\left(h_{1}, \ldots, h_{i-1}, \infty, h_{i+1}, \ldots, h_{k}\right) \notin\{\infty,-\infty, \Phi\}$ then for each $i, 1 \leq i \leq k, P_{i}$ is a closed flat expression not containing either of $\infty,-\infty$, or $\Phi$ as a subexpression. In other words the $P_{i}$ can be written as integer expressions over the signature of rings (i.e. $0,1, .,+,-$ ).

Proof. For simplicity of notation we take $k=2$, the other cases follow in the same manner. Suppose otherwise, and assume that $x_{1}$ occurs in $P_{i}$ or that any of $\infty,-\infty$ and of $\Phi$ occurs in $P_{i}$. We substitute $\infty$ for $x_{1}$ and we choose $h_{2}$ as in the statement of the Proposition. Substituting $h_{2}$ for $x_{2}$ we find with Proposition 1.1 that $P_{i}\left(\infty, h_{2}\right) \in\{\infty,-\infty, \Phi\}$. As a consequence $R\left(\infty, h_{2}\right) \in\{\infty,-\infty, \Phi\}$ which contradicts the assumption about $h_{2}$ that $R\left(\infty, h_{2}\right) \notin\{\infty,-\infty, \Phi\}$. The argument for the case that $x_{2}$ occurs in $P_{i}\left(x_{1}, x_{2}\right)$ is symmetric.

\section{Negative results on universality}

A format for expressions is called universal relative to $\mathbb{Q}_{ \pm \infty, \Phi}$ if for each expression $P$ there is an expression $Q$ in the given format so that $\mathbb{Q}_{ \pm \infty, \Phi} \models P=Q$. We do not formally define the notion of a format for expressions, instead we will merely consider some formats as examples. We focus on sums of simple fractions because that is a universal format in the case of meadows. We will first prove with two different examples that sums of simple fractions are not universal for transrational arithmetic.

\subsection{Sums of simple fractions are not universal}

In this section we will prove that sums of simple fractions do not constitute a universal format for open expressions of transrational arithmetic. The proof carries over to transreal arithmetic without modification. 
Theorem 2.1. Consider the following fraction $P$

$$
P=\frac{1}{\frac{1}{x}+\frac{1}{y}}
$$

There is no expression $R$ of the form $R \equiv P_{1} / Q_{1}+\ldots+P_{n} / Q_{n}$ with all $P_{i}$ and $Q_{i}$ flat forms such that $\mathbb{Q}_{ \pm \infty, \Phi} \models P=R$. In other words, in the context of transrational arithmetic, $P$ cannot be written as a sum of simple fractions.

Proof. We notice that on non- $\Phi$ arguments $P$ produces non- $\Phi$ values, in particular $P(1, \infty)=P(\infty, 1)=1 /((0 / \infty)+(1 / 1))=1 /(0+1)=1$, and also $P(1,-1)=P(\infty, \infty)=P(\infty,-\infty)=P(-\infty,-\infty)=\infty$ and $P(0,0)=0$. We assume that for all $x, y \in|\mathbb{Q}| \cup\{\infty,-\infty, \Phi\}, P(x, y)=R(x, y)$ with $R(x, y) \equiv$ $P_{1}(x, y) / Q_{1}(x, y)+\ldots+P_{n}(x, y) / Q_{n}(x, y)$ with the $P_{i}$ and the $Q_{i}$ flat forms, and from this assumption we will derive a contradiction. We first notice, using Proposition 1.3 and $P(1, \infty)=P(\infty, 1)=1$, that the $P_{i}$ are closed flat forms without occurrences of $\infty,-\infty$ and $\Phi$, so that the $P_{i}$ do not contain occurrences of either $x$ or $y$ and can be written as integer expressions with $0,1,+,-$ and .

Now consider $Q_{i}(x, y)$ with $i \in\{1, \ldots, n\}$, we will perform a case distinction on $Q_{i}(x, y)$ as to whether or not it is closed. First assume that $Q_{i}(x, y)$ is a closed expression, then it is equal to some expression say $t$ made up from $0,1,+,-, \cdot, \infty$, and $-\infty$. If $Q_{i}$ contains an occurrence of $\Phi$ the value of $R(x, y)$ is $\Phi$ for all substitutions and it can not be equal to $R(x, y)$ for all transrational values $x$ and $y$. If $\mathbb{Q}_{ \pm \infty, \Phi}=t=0$ then $P_{i} / Q_{i}$ is a closed term with a value in $\{\infty,-\infty, \Phi\}$. As a consequence the value of $R(x, y)$ is in $\{\infty,-\infty, \Phi\}$ for all substitutions for $x$ and $y$, for instance $x=y=1 / 2$. In the latter case, however one finds $P(1 / 2,1 / 2)=1$ which therefore differs from $R(1 / 2,1 / 2)$. It follows that $t$ has a value different from 0 , in which case $P_{i} / Q_{i}$ has a finite non- $\Phi$ value.

Now notice that at least one of the $Q_{i}(x, y)$ is not a closed flat form, as otherwise $R(x, y)$ is independent from its parameters $x$ and $y$ which is not the case for $P(x, y)$. Suppose that $x$ or $y$ (or both) occurs in $Q_{i}(x, y)$, then with Proposition $1.1 Q_{i}(\infty, \infty) \in\{\infty,-\infty, \Phi\}$. And as $P_{i}$ is a rational value $P_{i} / Q_{i}(\infty, \infty) \in$ $\{0, \Phi\}$. The case $\Phi$ can be excluded, because otherwise $R(\infty, \infty)=\Phi$ which differs from $P(\infty, \infty)=\infty$ and it follows that $P_{i}(\infty, \infty) / Q_{i}(\infty, \infty)=0$.

Taking these observations together it turns out that $R(\infty, \infty)$ equals a sum of terms each with a rational value, which may differ from 0 in case $Q_{i}$ is closed, and which must be equal to zero in case $Q_{i}$ is open. Therefore $R(\infty, \infty)$ equals a rational value which by necessity differs from $P(\infty, \infty)=\infty$, a contradiction which concludes the proof.

Proposition 2.1. Consider the following fraction $P$

$$
P=\frac{1}{x} \cdot \frac{1}{y}
$$

There is no expression $R$ of the form $R \equiv P_{1} / Q_{1}+\ldots+P_{n} / Q_{n}$ with all $P_{i}$ and $Q_{i}$ flat forms such that $\mathbb{Q}_{ \pm \infty, \Phi} \models P=R$. 
Proof. Assume that $P=R \equiv P_{1} / Q_{1}+\ldots+P_{n} / Q_{n}$. Because $P(1,1)=1 \neq \Phi$ none of the $P_{i}$ and $Q_{i}$ can contain $\Phi$. Moreover because $P(\infty, \infty)=0$, according to Proposition 1.3 none of the $P_{i}$ can contain $\infty$ or $-\infty$ and none of the $P_{i}$ can contain $x$ or $y$. It follows that the $P_{i}$ are closed integer expressions. As all closed fractions can be taken together into a single closed fraction we may assume that only $P_{n} / Q_{n}$ is a closed fraction and the other fractions are open. Suppose for some $i \in[1, \ldots, n-1], P_{i}=0$, then we know that $Q_{i}(\infty, \infty) \in\{\infty,-\infty, \Phi\}$ so that $P_{i} / Q_{i}(\infty, \infty)=\Phi$ and also $R(\infty, \infty)=\Phi$ which contradicts $P(\infty, \infty)=0$. Next we observe that for $i<n$ either all $P_{i}$ are positive or all $P_{i}$ are negative. Indeed if different signs occur, then, upon substituting $\infty$ for both variables, and assuming that no denominator takes the value $\Phi$ the result is a sum which includes a summand $\infty$ as well as a summand $-\infty$ so that the sum equals $\Phi$. Suppose that all $P_{i}$ are positive (the other case works in the same way), and consider $R(-1,0)$. We find that $R(-1,0)$ equals a sum of summands which are either rational values or $\infty$, as none of the summands can take value $-\infty$. Thus $R(-1, \infty)$ cannot be equal to $P(-1,0)=-\infty$ so that a contradiction is obtained which concludes the proof.

The following result literally corresponds to a result (Thm. 14 in [10]) about fractions in the meadow of rational numbers $\mathbb{Q}_{0}$ but the situation is rather different in the transrational case and the proof below is quite different from the proof given in [10] for the corresponding result concerning $\mathbb{Q}_{0}$.

Proposition 2.2. For all $n>0$, for the expression $P_{n}\left(x_{1}, \ldots, x_{n}\right) \equiv 1 / x_{1}+$ $\cdots+1 / x_{n}$ there is no expression $R_{n} \equiv Q_{1} / S_{1}+\ldots+Q_{n-1} / S_{n-1}$ with flat forms $Q_{i}$ and $S_{i}$ such that $\mathbb{Q}_{ \pm \infty, \Phi}=P_{n}\left(x_{1}, \ldots, x_{n}\right)=R_{n}\left(x_{1}, \ldots, x_{n}\right)$.

Proof. Suppose that $P_{n}\left(x_{1}, \ldots, x_{n}\right)=1 / x_{1}+\cdots+1 / x_{n}=R_{n}\left(x_{1}, \ldots, x_{n}\right)$ with $R_{n} \equiv Q_{1} / S_{1}+\ldots+Q_{n-1} / S_{n-1}$. We take 0 for the empty sum and then we may assume that $n>1$, because $1 / x_{1}=R_{1}(x)=0$ does not hold upon substituting 1 for $x_{1}$. So let $n>1$ be the smallest value for which a sum $R_{n}$ with said shape and properties can be found. We first notice that none of the $Q_{i}$ and $S_{i}$ may contain $\Phi$ as a subterm, because $P_{n}(\infty, \ldots, \infty)=0 \neq \Phi$. Further none of the $Q_{i}$ may contain $\infty$ or $-\infty$ as a subterm, because then $R_{n}(\infty, \ldots, \infty)=0$ cannot hold in view of Proposition 1.1. Further, using Proposition 1.3 we know that each of the $Q_{i}$ is closed because $R_{n}(\infty, \ldots, \infty)=P(\infty, \ldots, \infty)=0 \notin\{\infty,-\infty, \Phi\}$. For some $1 \leq i \leq n-1$ it must be the case that $x_{n}$ occurs in $S_{i}$, otherwise $R_{n}$ is independent from $x_{n}$ which is not the case for $P$ and therefore cannot be the case for $R_{n}$. Without loss of generality and perhaps after reordering the summands of $R_{n}$ we may assume that, $x_{n}$ occurs in $S_{n-1}$.

Substituting $\infty$ for $x_{n}$ we obtain $P_{n-1}\left(x_{1}, \ldots, n_{n-1}\right)=P_{n}\left(x_{1}, \ldots, n_{n-1}, \infty\right)$ $=\sum_{i=1}^{n-2}\left(Q_{i} / S_{i}\left(x_{1}, \ldots, x_{n-1}, \infty\right)\right)+Q_{n-1} / S_{n-1}\left(x_{1}, \ldots, x_{n-1}, \infty\right)$. Now using Proposition 1.1 for all $x_{1}, \ldots, x_{n-1}, S_{n-1}\left(x_{1}, \ldots, x_{n-1}, \infty\right) \in\{\infty,-\infty, \Phi\}$ from which it follows that $P_{n-1} / S_{n-1}\left(x_{1}, \ldots, x_{n-1}, \infty\right) \in\{0, \Phi\}$ for all $x_{1}, \ldots, x_{n-1}$, and moreover the value $\Phi$ is found only if at least one of $x_{1}, \ldots, x_{n-1}$ is set to $\Phi$. Each variable $x_{j}$ among $x_{1}, \ldots, x_{n-1}$ must occur in at least one of the flat forms $S_{1}, \ldots, S_{n-1}$ because otherwise for all non- $\Phi x_{1}, \ldots, x_{n-1}, \Sigma_{i=1}^{n-1} 1 / x_{i}=$ 
$\sum_{i=1}^{n-2}\left(Q_{i} / S_{i}\left(x_{1}, \ldots, x_{n-1}, \infty\right)\right)$ while $x_{j}$ does not occur in the RHS, which is not possible. Further, whenever, $Q_{n-1} / S_{n-1}\left(x_{1}, \ldots, x_{n-1}, \infty\right)$ takes value $\Phi$ that is the case already for $\sum_{i=1}^{n-2}\left(Q_{i} / S_{i}\left(x_{1}, \ldots, x_{n-1}, \infty\right)\right)$ so that for all $x_{1}, \ldots, x_{n-1}$ $P_{n-1}\left(x_{1}, \ldots, x_{n-1}\right)=\sum_{i=1}^{n-1} 1 / x_{i}=\sum_{i=1}^{n-2}\left(Q_{i} / S_{i}\left(x_{1}, \ldots, x_{n-1}, \infty\right)\right)$ which contradicts the minimality of $n$ as chosen above thereby concluding the proof.

\subsection{Mixed fractions are not universal for expressions with a single variable}

In [6] it is shown that in the meadow of rational numbers $\mathbb{Q}_{0}^{d}$ each expression with a single variable can be written as a mixed fraction, that is a sum $P+R / S$ of a flat form $P$ and a simple fraction $R / S$. A corresponding result does not apply to transrational arithmetic, however. We will demonstrate two examples of expressions with a single variable which cannot be written as a mixed fraction. First consider another simplifying transformation for fractions, which is easily checked.

Example 2.1.

$$
\frac{1}{x}+\frac{1}{1-x}=\frac{1}{x \cdot(1-x)}
$$

Theorem 2.2. Consider the following expression P:

$$
P=\frac{1}{x}-\frac{1}{1-x}
$$

There is no expression $R$ of the form $R \equiv Q+S / T$ where $Q, S$, and $T$ are flat forms, such that $\mathbb{Q}_{ \pm \infty, \Phi} \models P=R$. In other words, in the context of transrational arithmetic, $P$ cannot be written as a mixed fraction.

Proof. To begin with we notice that it is not the case that transrational arithmetic satisfies the equation $(-x) /(-y)=x / y$, and in particular $(-1) /(-0)=$ $(-1) \cdot \infty=-\infty \neq \infty=1 / 0$. This remark explains the difference with example 2.1. Now suppose that $P(x)=Q(x)+S(x) / T(x)$ with $Q, S$, and $T$ flat forms. If $x$ occurs in $P(x)$ then by Proposition 1.1 one finds that $R(\infty) \in\{\infty,-\infty, \Phi\}$ which contradicts $P(\infty)=0$. With Proposition 1.3 one finds that $S(x)$ is closed. So $P(x)=q+s / T(x)$ where $q$ and $s$ are closed integer expressions in which $\infty,-\infty$, and $\Phi$ do not occur. Now $q+s / T(x)$ can only take the value $\infty$ or take the value $-\infty$ if $T(x)=0$, so we find that both $T(0)=0$ and $T(1)=0$. But then $T(0)=T(1)$ and therefore $P(1)=q+s / T(0)=q+s / T(1)=P(0)$. This observation, however, contradicts the fact that $P(0)=\infty$ and $P(1)=-\infty$, thereby completing the proof.

Theorem 2.3. Consider the following expression P:

$$
P=\frac{1}{x}+\frac{1}{x-1}+\frac{1}{x-2}
$$

There is no expression $R$ of the form $R \equiv Q+S / T$ where $Q, S$, and $T$ are flat forms, such that $\mathbb{Q}_{ \pm \infty, \Phi} \models P=R$. In other words, in the context of transrational arithmetic, $P$ cannot be written as a simple fraction. 
Proof. Suppose that $P=R$ with $R \equiv Q+S / T$. As in the proof of Theorem 2.2 we find that $R$ and $S$ are closed and do not contain $\infty,-\infty$ and $\Phi$. So these terms are closed integer expressions, say $s$ and $r$. Next we note that $x$ must occur in $T$ as otherwise $R$ is independent from $x$ which is not the case for $P$, however. It follows that $T(\infty) \in\{\infty,-\infty, \Phi\} . T(\infty)=\Phi$ is impossible because then $R(\infty)=\Phi$ while $P(\infty)=0$. Both other cases remain. If $T(\infty)=\infty$ then $0=P(\infty)=q+s / \infty=q+s \cdot 0=q$. The same conclusion that $q=0$ also follows if $T(\infty)=-\infty$. It follows that $q=0$ and $R=s / T$. Now $P(0)=P(1)=P(2)=$ $\infty$, so $R(0)=R(1)=R(2)=\infty$. This implies that $T(0)=T(1)=T(2)=0$. As $T(x)$ depends on $x$, it must be the case that $T(x)$ is equivalent to a nontrivial polynomial say of degree $k \geq 1$, so that $T$ having three roots at least, $T$ is (equal to) a polynomial of degree 3 at least, i.e. $d g(T)=k \geq 3$. Now notice that except for $x \in\{0,1,2\}$,

$P(x)=\frac{(x-1) \cdot(x-2)+x \cdot(x-2)+x \cdot(x-1)}{x \cdot(x-1) \cdot(x-2)}=\frac{3 \cdot x \cdot x-6 \cdot x+2}{x \cdot(x-1) \cdot(x-2)} \equiv \frac{A(x)}{B(x)}$

We have $d g(A)=2$ and $d g(B)=3$, moreover almost everywhere $A(x) / B(x)=$ $s / T(x)$ and, therefore almost everywhere $A(x) \cdot T(x)=B(x)$, whence for all rational $x, A(x) \cdot T(x)=B(x)$. It follows that $d g(A)+d g(T)=d g(B)$, which contradicts $d g(A)=2, d g(T) \geq 3$ and $d g(B)=3$.

Problem 2.1. Are sums of simple fractions universal for expressions involving a single variable (relative to $\mathbb{Q}_{ \pm \infty, \Phi}$ )?

\section{Division depth and fraction hierarchy}

For expressions we define the division depth $d$ / inductively as follows:

$d_{/}(0)=d_{/}(1)=d_{/}(\infty)=d_{/}(-\infty)=d_{/}(\Phi)=0$,

$d_{/}(-x)=d_{/}(x), d_{/}(x+y)=d_{/}(x-y)=\max \left(d_{/}(x), d_{/}(y)\right)$,

$d_{/}\left(x^{-1}\right)=d_{/}(x)+1, d_{/}(x \cdot y)=d_{/}(x / y)=\max \left(d_{/}(x), d_{/}(y)\right)+1$.

The following question we will refer to as the fraction hierarchy problem for $\mathbb{Q}_{ \pm \infty, \Phi}$. This question is left open.

Problem 3.1. Is there a bound $k_{f r}$ such that for each expression $P$ there is an expression $Q$ with $d_{/}(Q)<k_{\text {fr }}$ such that $\mathbb{Q}_{ \pm \infty, \Phi} \models P=Q$ ?

For $\mathbb{Q}_{0}$ the fraction hierarchy problem has a positive solution and expressions with fraction depth 1 suffice to express all expressions modulo provable equality. For $\mathbb{Q}_{ \pm \infty, \Phi}$, we expect that the fraction hierarchy problem has a negative solution but we have not been able to prove that conjecture.

\section{Concluding remarks}

If division is taken for a partial function, the conventional viewpoint, or if division on zero is supposed to return an absorptive value that propagates though 
all function symbols, the simplest totalised version of the conventional viewpoint, thereby representing one way to model the conventional viewpoint on division by zero, the topic of fraction theory trivialises because all expressions are equivalent to a simple fraction.

For other total versions of arithmetic a theory of fractions emerges which is an intriguing subject of its own and which reveals significant differences for the different options of arithmetical datatypes. We have made some progress on fraction theory for transrational arithmetic thereby highlighting the contrasts with properties of fractions in the meadow or rational numbers. Various questions are left open, including analysing relations with the fraction theory of the wheel of rational numbers as introduced in [23] and studied in [15] and [16]. We notice that [12] investigates a different aspect of a theory of fractions by providing a notion of division safe calculation for meadows with divisive notation. Similar ideas may be of relevance for transrational arithmetic, and merit

further exploration. Finally we mention that [5] provides yet another aspect of fraction theory for meadows by casting reasoning about fractions as an instance of working with a paraconsistent logic.

\section{References}

[1] J.A.D.W. Anderson, N. Völker, and A. A. Adams. Exact numerical computation of the rational general linear transformations. Vision Geometry XI, eds. J. Latecki, D. M. Mount and A. Y. Wu, Proceedings of SPIE Vol. 4794, 22-28 (2002), http://www.bookof paragon.com/Mathematics/ SPIE. 2002.Exact.pdf.

[2] J.A. Anderson, N. Völker, and A. A. Adams. Perspecx Machine VIII, axioms of transreal arithmetic. Vision Geometry XV, eds. J. Latecki, D. M. Mount and A. Y. Wu, 649902; https://doi.org/10.1117/12.698153 (2007).

[3] P. Benacerraf. What numbers could not be. The Philosophical Review. 74 (1), 47-73 (1965).

[4] J.A. Bergstra. Division by Zero, a Survey of Options. Transmathematica https://doi.org/10.36285/tm.v0i0.17, (2019).

[5] J.A. Bergstra, I. Bethke. Note on paraconsistency and reasoning about fractions. J. of Applied Non-Classical Logics. http://dx.doi.org/10. 1080/11663081.2015.1047232 (2015). (Also https://arxiv.org/abs/ 1410.8692.)

[6] J. A. Bergstra, I. Bethke, and D. Hendriks. Universality of mixed fractions in divisive meadows. https://arxiv.org/abs/1707.00499.

[7] J. A. Bergstra, I. Bethke, and A. Ponse. Cancellation meadows: a generic basis theorem and some applications. The Computer Journal, 56 (1): 3-14, (2013). (Also https://arxiv.org/abs/0803.3969.) 
[8] J.A. Bergstra, Y. Hirshfeld, and J.V. Tucker. Meadows and the equational specification of division. Theoretical Computer Science, 410 (12), 1261-1271 (2009).

[9] J.A. Bergstra and C.A. Middelburg. Inversive meadows and divisive meadows. Journal of Applied Logic, 9 (3): 203-220 (2011). (Also https: //arxiv.org/abs/0907.0540.)

[10] J.A. Bergstra and C.A. Middelburg. Transformation of fractions into simple fractions in divisive meadows. Journal of Applied Logic, 16, 92-110. (2016). (Also https://arxiv.org/abs/1510.06233.)

[11] J.A. Bergstra and A. Ponse. Division by zero in common meadows. In $R$. de Nicola and R. Hennicker (editors), Software, Services, and Systems (Wirsing Festschrift), LNCS 8950, pages 46-61, Springer, (2015). (Also https://arxiv.org/abs/1406.6878.)

[12] J.A. Bergstra and J.V. Tucker. Division safe calculation in totalized fields. Theory of Computing Systems, 43, 410-424 (2008).

[13] I. Bethke and P.H. Rodenburg. The initial meadows. Journal of Symbolic Logic, 75 (3), 888-895, (2010).

[14] H. D. Ehrich, M. Wolf, and J. Loeckx. Specification of Abstract Data Types. Vieweg + Teubner, ISBN-10:3519021153, (1997)

[15] J. Carlström. Wheels-on division by zero. Math. Structures in Computer Science, 14 (1) pp. 143-184, (2004).

[16] J. Carlström. Partiality and choice, foundational contributions. PhD. Thesis, Stockholm University, http://www.diva-portal.org/smash/get/ diva2:194366/FULLTEXT01.pdf, (2005).

[17] P. Hilton. What numbers are not, a philosophy of mathematics essay about the problem of trying to define numbers rigorously. https://hilton.org. uk/what_numbers_are_not.html (consulted May 25, 2019).

[18] Y. Komori. Free algebras over all fields and pseudo-fields. Report 10, pp. 9-15, Faculty of Science, Shizuoka University (1975).

[19] H. Michiwaki, S. Saitoh, and N. Yamada. Reality of the division by zero $z / 0=0$. International Journal of Applied Physics and mathematics, vol. 6 no. 1 (2016). http://www.ijapm.org/show-63-504-1.html, https:// doi.org/10.17706/IJAPM (2016).

[20] J-. F. Nicaud, D. Bouhineau, and J-. M. Gelis. Syntax and semantics in algebra. Proc. 12th ICMI Study Conference, The University of Melbourne, 2001. HAL archives-ouvertes https://hal.archives-ouvertes. fr/hal-00962023/document, (2001). 
[21] T.S. dos Reis and J.A.D.W. Anderson. Construction of the transcomplex numbers from the complex numbers. Proc. WCECS 2014, http://www. iaeng . org/publication/WCECS2014/WCECS2014_pp97-102.pdf, (2014).

[22] H. Ono. Equational theories and universal theories of fields. Journal of the Mathematical Society of Japan, 35(2), 289-306 (1983).

[23] A. Setzer. Wheels (draft). http://www.cs.swan.ac.uk/ csetzer/ articles/wheel.pdf, (1997). 